\title{
Study of the Static Properties I-V of Mosfet
}

\author{
Ouassila Benzaoui ${ }^{1,3}$ and Cherifa Azizi ${ }^{2,3}$ \\ 1. Technology Department, Faculty of Technology, 20 August 1955 University, BP 26, Skikda 21000, Algeria \\ 2. Sciences Institute, Larbi Ben M'hidi University, BP 358, Constantine Street, Oum El Bouaghi 04000, Algeria \\ 3. Department of Physics, Faculty of Science, Thin Films and Interfaces Laboratory, Mentouri University, Constantine 25000, \\ Algeria
}

Received: May 03, 2013 / Accepted: June 06, 2013 / Published: August 25, 2013.

\begin{abstract}
Today, telecommunications, data processing, physics and electronics, take a very important place in the activities of research of the various laboratories. In the field of the ultra high frequencies, the field-effect transistor MOSFET caused many studies and research to exploit its interesting and promising characteristics as well as possible. The objective of this contribution is devoted to study the static properties I-V of MOSFET. The study enables us to calculate the drain current as function of bias in both linear and saturated modes; this effect is evaluated using a numerical simulation program, one could notice that the MOS transistor characteristics are very sensitive to the temperature. The load of inversion via the threshold voltage and the mobility of the carriers are the two principal impacted parameters, it was noted that the increase in the temperature induces a drop of the threshold voltage like that of mobility, and an immediate consequence of this reduction is the diminution in the drain current. One can thus conclude that the temperature influences the performances of the device; more it is low, better is the reliability of the device under operation.
\end{abstract}

Key words: MOSFET, static proprieties, performances.

\section{Nomenclature}

$L: \quad$ Grid length (distance source-drain)

$Z$ : $\quad$ Grid width

$d: \quad$ Oxide thickness

$I_{D}: \quad$ Drain current

$I_{D S A T}: \quad$ Drain current saturation

$V_{D}: \quad$ Drain voltage

$V_{D S A T}: \quad$ Drain voltage saturation

$V_{G}: \quad$ Grid voltage

$V_{T}: \quad$ Threshold voltage

$e: \quad$ Electron charge

$W: \quad$ Limit the space charge zone

$N_{a}$ : Concentration of dopants in the channel

$C_{o x}$ : $\quad$ Oxide capacity

$E: \quad$ Electric field

$E_{c}$ : $\quad$ Critical electric field (measured experimentally parameter $\mathrm{E}_{\mathrm{c}}$ is about $5 \times 10^{4} \mathrm{v} / \mathrm{cm}$ )

$T: \quad$ The temperature of the network

$T_{0}: \quad$ The room temperature (300k)

$K: \quad$ Factor varying between (2.2 and 2.7)

Corresponding author: Ouassila Benzaoui, assistant professor, research field: field effect transistor (MESFET, MOSFET). E-mail: o_benzaoui@yahoo.fr, o.benzaoui@gmail.com.

\section{Greek Letters}

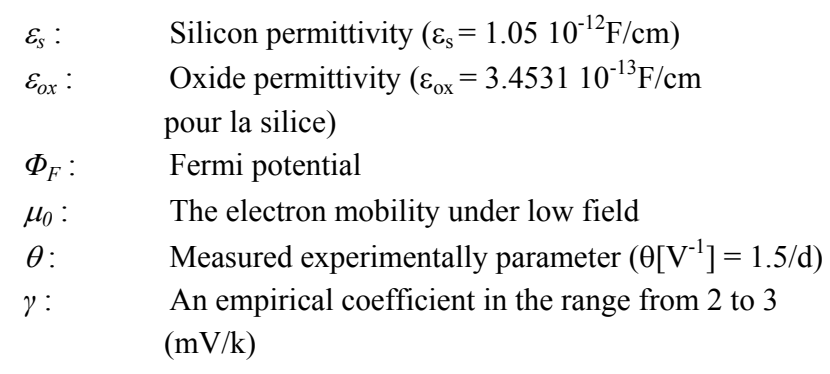

\section{Introduction}

The MOS transistor is by far, the device the most encountered in the current production of semiconductor components, several acronyms are used in the literature to describe the metal oxide semiconductor (MOS) transistor: MOS field effect transistor (MOSFET), insulated gate field effect transistor (IGFET) and metal oxide semiconductor transistor (MOST) [1].

Silicon technologies field-effect MOSFET transistors are very mature and offer components with very honorable performances because of the intrinsic 
physical properties at relatively low cost. This remains a major asset in the current context making it possible to fulfill the requirements of communication systems in terms of power.

The transistors are traditionally modulated by using the model of the equivalent diagram. But this kind of models can only give one limited outline on the physical behavior of the component, that is why the physical models based on analytical description intervene, in terms of transport properties, geometrical and technological parameters of the transistor.

\section{Calculation of the Drain Current}

Fig. 1 represents the elementary structure of the studied device; it is a $\mathrm{N}$ type MOSFET working in the static regime, its modeling requires several simplifications due to the difficulties imposed by the avalanche phenomena (substrate-drain junction) and source-drain drilling [2].

To calculate the drain current of the MOSFET one adopts the following assumptions [3]:

- The insulator $\left(\mathrm{SiO}_{2}\right)$ is ideal: absence of charge traps in insulator and at the interface of the semiconductor. There is no difference in work between metal and the semiconductor;

- The mobility of the carriers is constant in the inversion layer;

- The doping of the channel is uniform in all the substrate;

- The leakage current is negligible.

Approximation of the gradual charge: The transverse field $E_{x}$ in the channel is more important than the longitudinal field $\mathrm{E}_{\mathrm{y}}$.

Thus the variation of the drain current $I_{D}$ with the drain-source voltage $V_{D}$ and the gate-source voltage $V_{G}$ is given by the following general relation [4]:

$I_{D}=\frac{Z \mu C_{o x}}{L}\left(\left(V_{G}-\frac{V_{D}}{2}-2 \phi_{F i}\right) V_{D}-\frac{2}{3} \frac{\left(2 e N_{a} \varepsilon_{S}\right)^{1 / 2}}{C_{o x}}\left(\left(V_{D}+2 \phi_{F i}\right)^{3 / 2}-\left(2 \phi_{F i}\right)^{3 / 2}\right)\right)(1)$

However, this equation is rewritten differently, according to the operation regime of the transistor which depends primarily on the value of the drain voltage $\mathrm{V}_{\mathrm{D}}$.

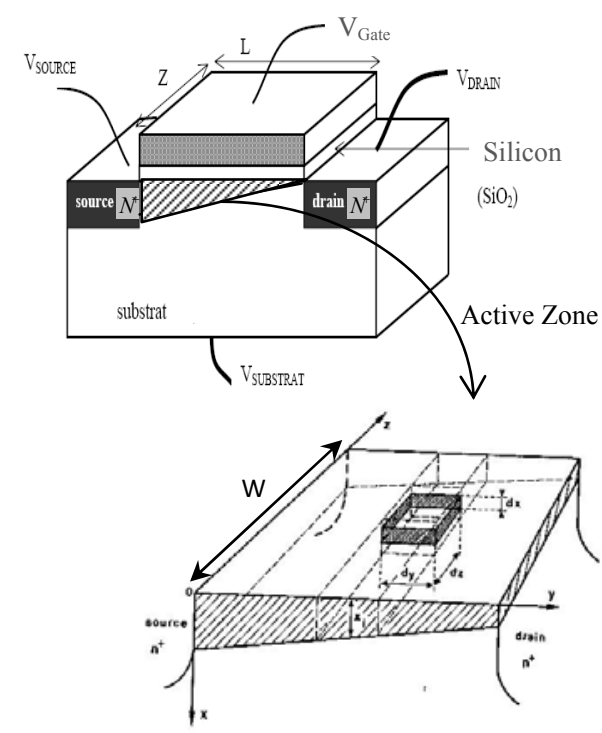

Fig. 1 Structure of the MOSFET type N.

\subsection{Linear regime}

The drain voltage in this regime obeys to the following condition: $\mathrm{V}_{\mathrm{D}} \leq \mathrm{V}_{\mathrm{G}}-\mathrm{V}_{\mathrm{T}}$.

Thus, the equation of drain current is rewritten [5]:

$$
I_{D}=\frac{Z \mu C_{o x}}{L}\left(V_{G}-V_{T}\right) V_{D}
$$

With $\mathrm{V}_{\mathrm{T}}$ is the threshold voltage and $\mathrm{C}_{\mathrm{ox}}$ is the insulator capacitance, both are given by:

$$
\begin{gathered}
V_{T}=2 \phi_{F i}+\left(4 e N_{a} \varepsilon_{s} \phi_{F i}\right)^{1 / 2} / C_{o x} \\
C_{o x}=\frac{\varepsilon_{o x}}{d}
\end{gathered}
$$

\subsection{Saturation Regime}

The drain voltage is conditioned by: $\mathrm{V}_{\mathrm{D}}>\mathrm{V}_{\mathrm{G}}-\mathrm{V}_{\mathrm{T}}$

The drain current becomes [5]:

$$
I_{\text {Dsat }}=\frac{Z \mu C_{o x}}{2 L}\left(V_{G}-V_{T}\right)^{2}=\frac{Z \mu C_{o x}}{2 L} V_{D s a t}^{2}
$$

Where $\mathrm{V}_{\text {Dsat }}$ is the saturation voltage given by:

$$
V_{\text {Dsat }}=V_{G}-2 \phi_{F i}=V_{G}-V_{T}
$$

\section{Results and Discussion}

For the numerical calculation of the drain current versus bias voltage, one uses the data of transistors MOSFET1 $(L=2.5 \mu \mathrm{m})$; MOSFET2 $(L=1.5 \mu \mathrm{m})$ whose parameters are given in Table 1. 
Table 1 Parameters of MOSFET.

\begin{tabular}{lcccccc}
\hline MOSFET & $\begin{array}{c}\mathrm{L} \\
(\mu \mathrm{m})\end{array}$ & $\begin{array}{c}\mathrm{D} \\
\left(\mathrm{A}^{\circ}\right)\end{array}$ & $\begin{array}{c}\mathrm{Z} \\
(\mathrm{cm})\end{array}$ & $\begin{array}{c}\mathrm{N}_{\mathrm{a}} \\
\left(\mathrm{cm}^{-3}\right)\end{array}$ & $\begin{array}{c}\mu_{0} \\
\left(\mathrm{~cm}^{2} \cdot \mathrm{s}^{-1} \cdot \mathrm{v}^{-1}\right)\end{array}$ & $\begin{array}{c}\Phi_{\mathrm{Fi}} \\
(\mathrm{V})\end{array}$ \\
\hline MOSFET1 & 1.5 & 1,500 & 3.53 & $10^{16}$ & 277 & 0.35 \\
MOSFET2 & 2.5 & 1,000 & 2.81 & $10^{16}$ & 360 & 0.35 \\
\hline
\end{tabular}

The Figs. 2 and 3 represent the variation of the drain current $I_{D}$ versus $V_{D}$ for various values of $V_{G}$ obtained respectively for the MOSFET1 $(\mathrm{L}=1.5 \mu \mathrm{m})$ and the MOSFET2 $(L=2.5 \mu \mathrm{m})$, one notes that the drain current $I_{D}$ increases with the voltage $\mathrm{V}_{\mathrm{D}}$, then it saturates in the case of the MOSFET2 for a value for which the Space Charge Region (S.C.R) covers all the channel, contrary to the MOSFET1 which is saturated before the channel pinch. This is due to the fact that the length of the channel is short, which leads quickly to appreciable electric field values and consequently, fast saturation of the carriers velocity in the channel.

This means that the saturation of the drain current, in this case, is related to the saturation of the carriers velocity. It should be also noted that $I_{D}$ undergoes a reduction until its annulment for a nonzero value of $\mathrm{V}_{\mathrm{G}}$ known as threshold voltage.

\section{Effect of Variable Mobility}

The mobility is a physical quantity defined as the proportionality factor between carriers velocity and the electric field, it depends in a complex way, on the nature and the frequency of the collisions and interactions undergone by the carriers during their displacement, and it has a great influence on the current-voltage characteristics [5].

To show this dependence the two following laws (Eq. (7) [4] and Eq. (8) [1]) were chosen:

$$
\begin{gathered}
\mu_{1}=\frac{\mu_{0}}{1+\theta\left(V_{G}-V_{T}\right)+E / E_{c}} \\
\mu_{2}=\frac{\mu_{0}}{\left[1+\theta\left(V_{G}-V_{T}\right)\right]\left[1+E / E_{c}\right]}
\end{gathered}
$$

$\mu_{0}$ is the electron mobility under low field, $\theta$ and $E_{c}$ (critical field) are two parameters experimentally measured $\left(\theta\left[\mathrm{V}^{-1}\right]=1.5 / \mathrm{d}, \mathrm{E}_{\mathrm{c}}\right.$ is about $\left.5 \times 10^{4} \mathrm{v} / \mathrm{cm}\right)$.
In fact, the use of these expressions for MOSFET1 transistor enables us to obtain the Fig. 4 and 5 on

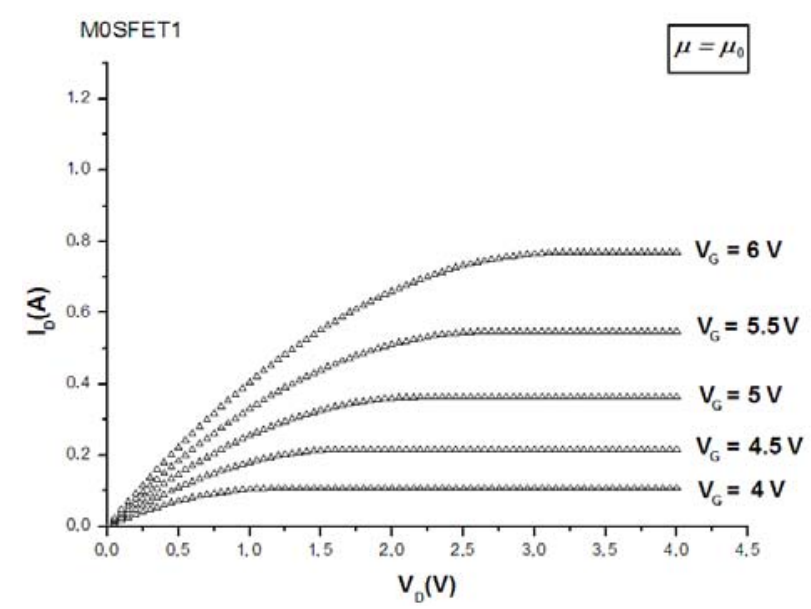

Fig. 2 Variation of $I_{D}$ versus $V_{D}$ for various $V_{G}$ values for MOSFET1 transistor.

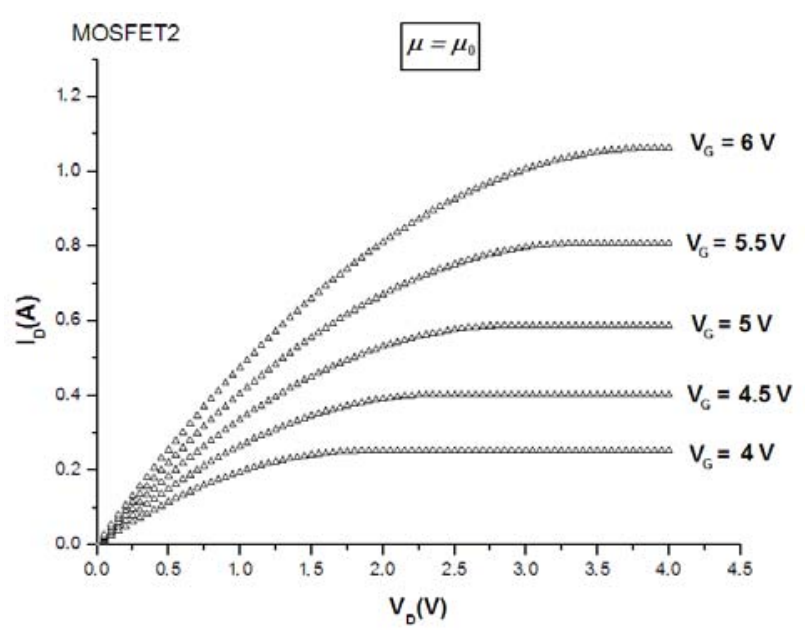

Fig. 3 Variation of $I_{D}$ versus $V_{D}$ for various $V_{G}$ values for MOSFET2 transistor.

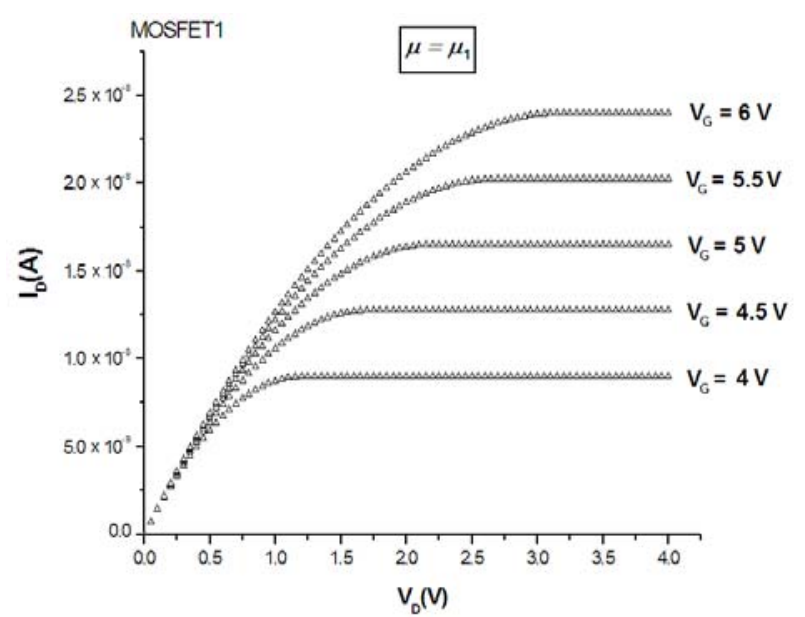

Fig. 4 Variation of $I_{D}$ versus $V_{D}$ for various $V_{G}$ values obtained by using the expression of the mobility $\mu_{1}$. 


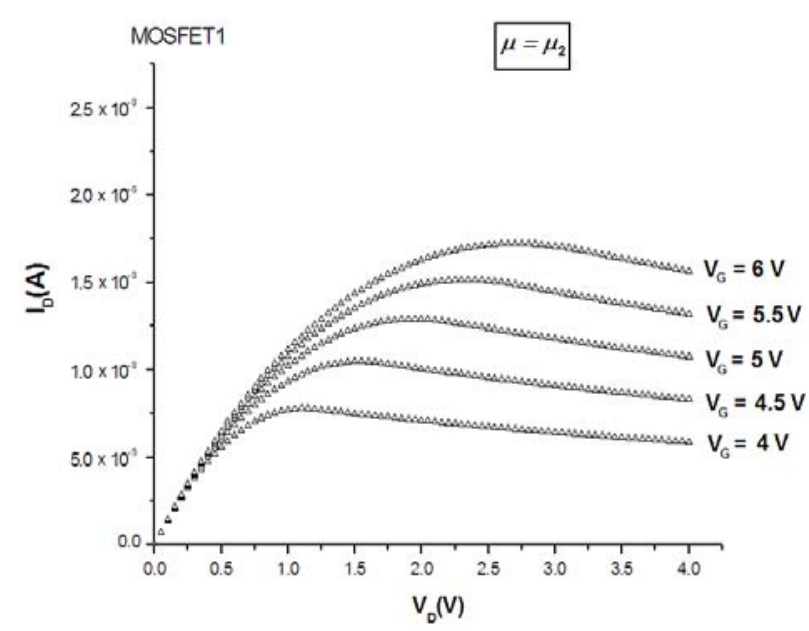

Fig. 5 Variation of $I_{D}$ versus $V_{D}$ for various $V_{G}$ values obtained by using the expression of the mobility $\mu_{2}$.

which one notes that the variations of the drain current are maximum in the case of $\mu_{1}$. The current undergoes a reduction in the saturation regime which is remarkable in the case of the mobility $\mu_{2}$. This decrease is due to the fact that the accumulation phenomenon of the charge carriers occurring in the channel is not taken into account.

\section{Effect of the Temperature}

The current characteristics of the MOSFET are strongly related to the temperature. However the majority of simulations suppose that the temperature of the component is constant. The room temperature $(300 \mathrm{~K})$ is usually taken as the component temperature.

The dependence of the carriers mobility on the temperature plays a fundamental role in the determination of the current. A simple law to model the variation of mobility with the temperature is the following one [6-8]:

$$
\mu(T)=\mu\left(T_{0}\right)\left[\frac{T}{T_{0}}\right]^{-k}
$$

The variation the saturation speed with the temperature for the MOSFET is given by [9]:

$$
\mathrm{V}_{\mathrm{Sat}}=\frac{2.410^{5}}{1+0.8 \exp \left[\frac{\mathrm{T}}{600}\right]}
$$

The threshold voltage as a function of the temperature is given by [10-12]:

$$
V_{T}(T)=V_{T}\left(T_{0}\right)\left[1-\gamma\left(T-T_{0}\right)\right]
$$

where, $T$ is the temperature of the network, $T_{0}$ the room temperature $(300 \mathrm{~K}), \mathrm{K}$ a factor varying between 2.2 and 2.7 and $\gamma$ is an empirical coefficient in the range from 2 to $3 \mathrm{mV} / \mathrm{k}$.

We studied the effect of the temperature on the static characteristics of the component, by numerical simulation using the mobility laws, the saturation speed and the threshold voltage. Calculation was carried out for MOSFET1 transistor.

Fig. 6 presents the variation of the drain current $I_{D}$ versus $V_{D}$ for various temperatures. It can be seen that the drain current decreases with the temperature increase. This is an immediate consequence of the reduction of the mobility and the saturation speed as illustrated in Figs. 7 and 8, respectively.

It should be noted that the rise in the temperature induces an intensive interaction between charge carriers and the optical phonons of the network. This leads to the decrease of the mobility and a limitation of the saturation speed of the carriers for a critical value of the lateral field $E_{C}$. The latter constantly increases with the temperature (Fig. 9).

The Figs. 10 and 11 show I-V characteristics in the case of variable mobilities $\mu_{1}$ and $\mu_{2}$. The same explanation as in the case of constant mobility $\mu_{0}$ can

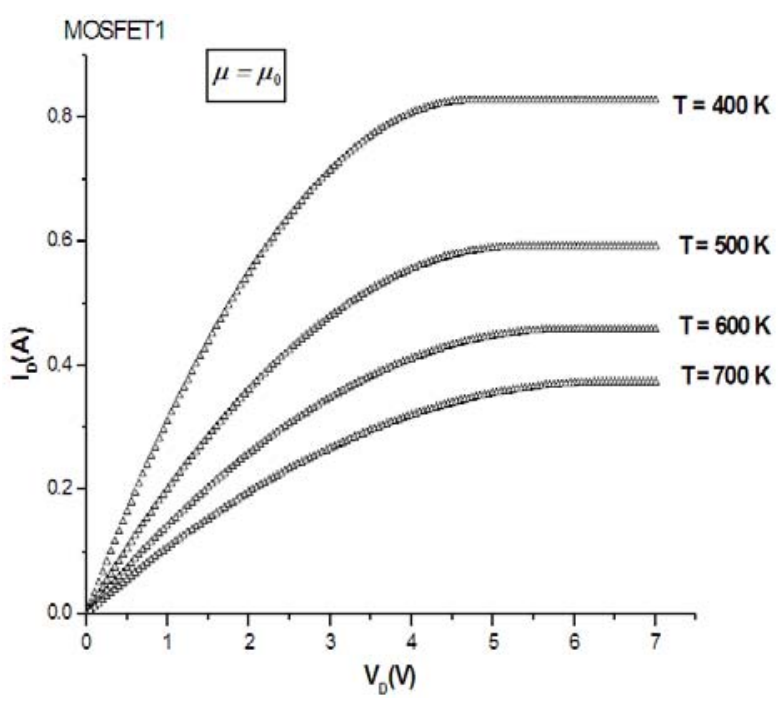

Fig. 6 Variation of $I_{D}$ versus $V_{D}$ for various. 


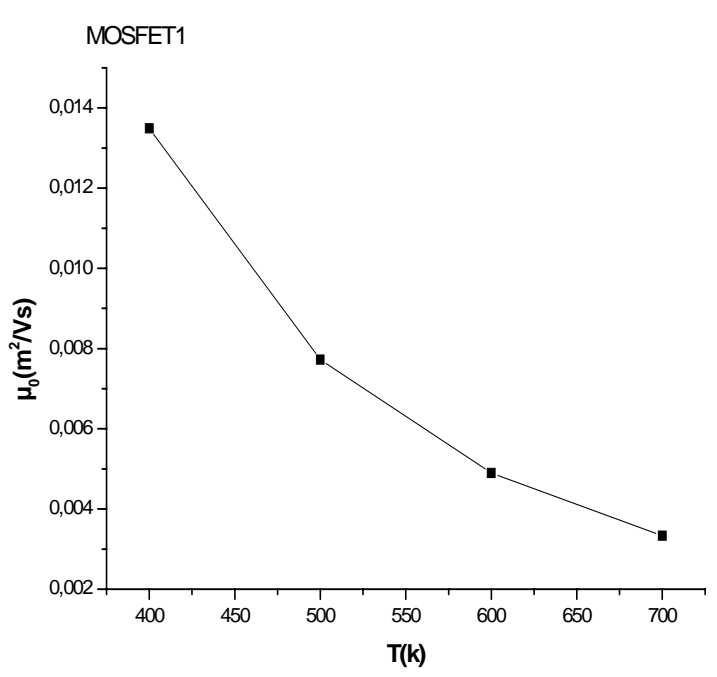

Fig. 7 Variation of mobility $\mu_{0}$ versus temperature.

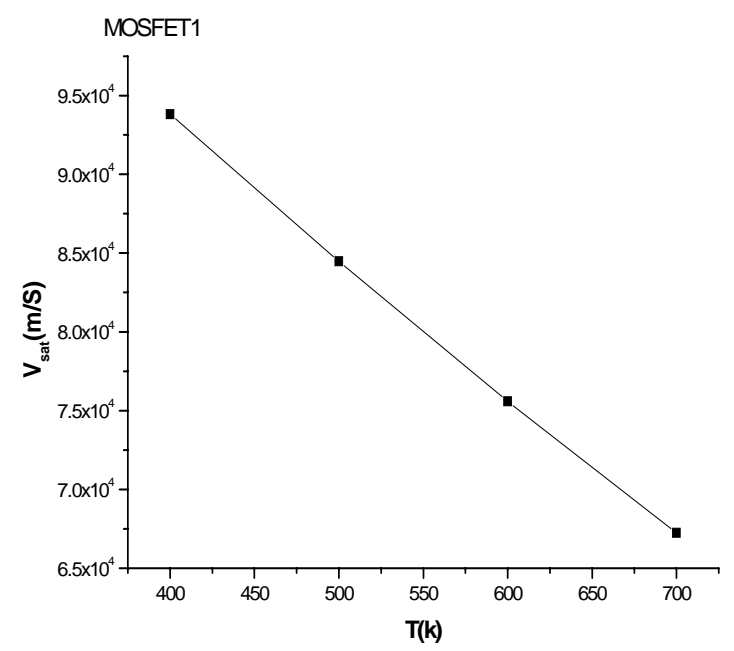

Fig. 8 Variation of saturation speed $V_{S a t}$ versus the temperature.

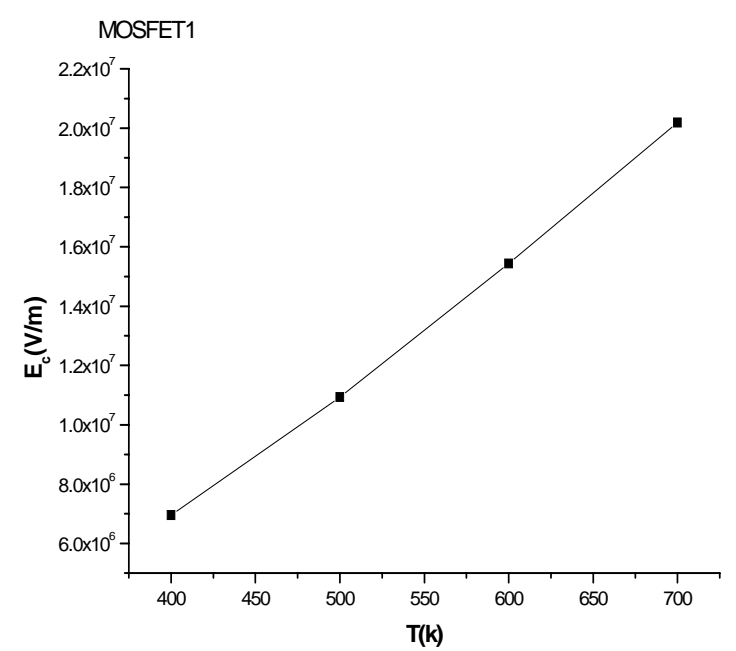

Fig. 9 Variation of the saturation of the electric field $E_{c}$ versus the temperature.

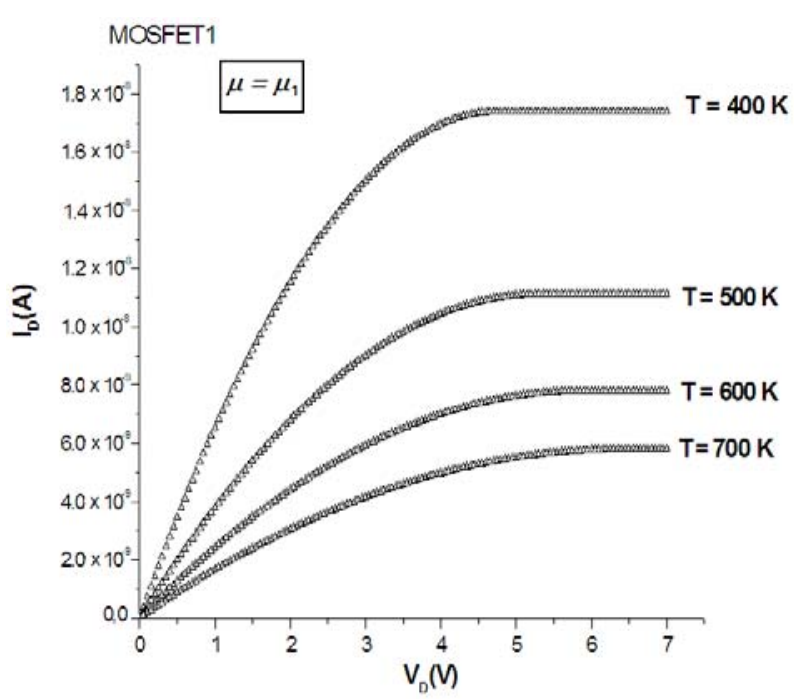

Fig. 10 Variation of $I_{D}$ versus $V_{D}$ for various temperatures values obtained by using the expression of the mobility $\mu_{1}$.

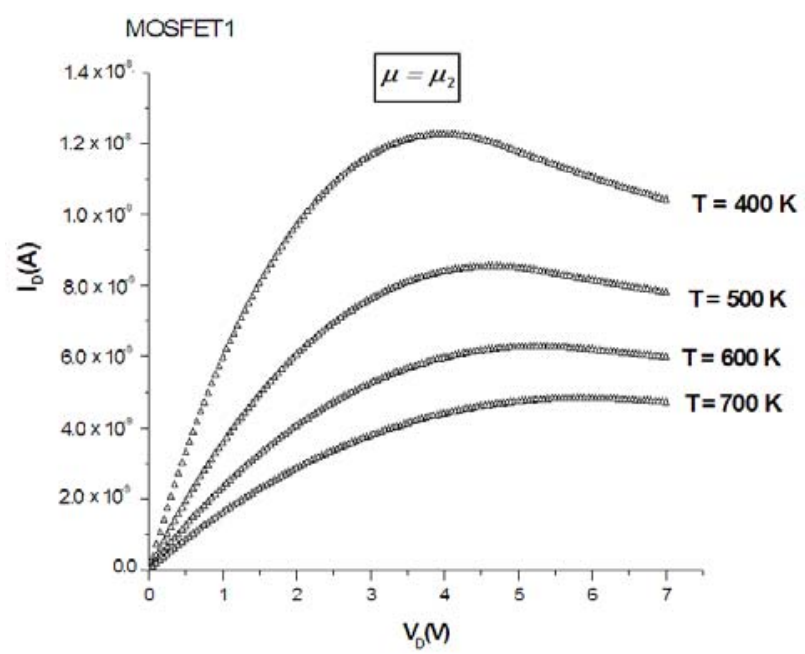

Fig. 11 Variation of $I_{D}$ versus $V_{D}$ for various temperatures values obtained by using the expression of the mobility $\mu_{2}$.

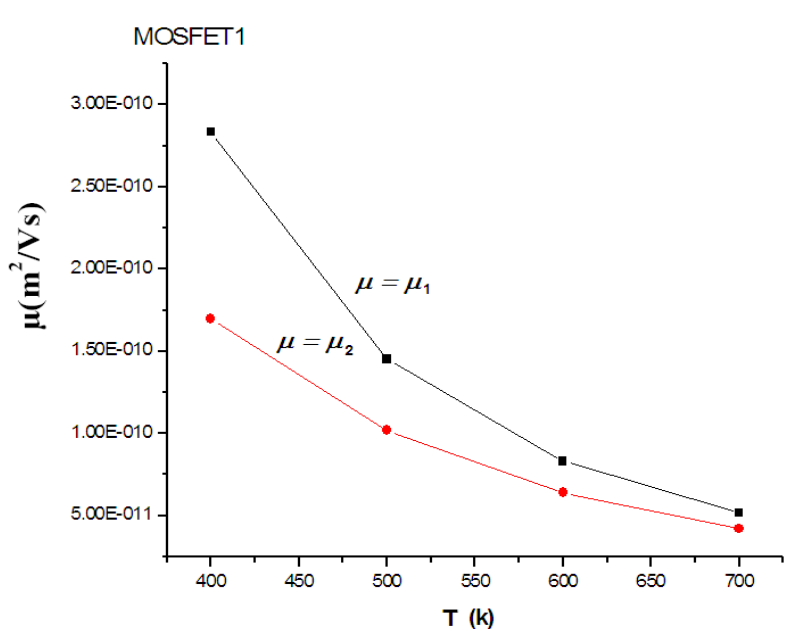

Fig. 12 Variation of $\mu_{1}$ and $\mu_{2}$ mobilities versus temperature. 
be given, i.e., the drain current is better at low temperature.

The variation of $\mu_{1}$ and $\mu_{2}$ mobilities versus the temperature are presented on the Fig. 12, they decrease as the temperature increases.

\section{Conclusions}

In this paper, we developed a model allowing the description of the static electric operation of MOSFET in linear and saturated regimes, by taking into account the variation of mobility versus the electric field. The mobility law in the MOSFET which gives maximum variations of the drain current is given by the Eq. (7).

In addition the variation of the $\mathrm{I}-\mathrm{V}$ characteristics of the component versus the temperature was obtained; the lower the temperature, the better is the reliability of the device under operation.

\section{References}

[1] T. Skotnicki, Transistor MOS and Manufacturing Engineering Technology, Electronics Treated, 1987. (in French)

[2] D. Muller, Optimization potential of a LDMOS transistor for integration of RF power amplifier on silicon, Ph.D. Thesis, University Limoge, 61-2006. (in French)

[3] S.M. SZE, Physics of Semiconductor Devices, 2nd ed.,
John Wiley \& Sons, Taipei, 1981, pp. 431-496.

[4] H. Mathieu, Physics of semiconductors and electronic components, 2nd Ed., Dunod, Paris, 2001. (in French)

[5] F. Morancho, The power MOS transistor trench: Modeling and Performance Limits, University Paul Sabatier, Toulouse, 1996, p. 96482. (in French)

[6] N.D. Arora, J.R. Hauser, D.J. Roulston, Electron and hole mobilities in silicon as a function of concentration and temperature, IEEE Transaction on Electron Devices 30 (6) (1983) 658-663.

[7] D.B.M. Klaassen, A united mobility model for device simulation: II. Temperature dependence of carrier mobility and lifetime, Solid-State Electronics 35 (1992) 961-967.

[8] J.M. Dorkel, P. Leturq, Carrier mobilities in silicon semi-empirically related to temperature, doping and injection level, Solid-State Electronics 4 821-824 (1981).

[9] C. Jacobini, C. Canali, G. Ottaviani, A. Alberigi Quaranta, A review of some charge transport properties of silicon, Solid-State Electronics 20 (1977) 77-89.

[10] N.D. Arora, MOSFET Models for VLSI Circuit Simulation-Theory and Practice, Editions Springer Verlag, 1993.

[11] B. Fatemizadeh, D. Silber, Modeling of LDMOST and LIGBT structures at high temperatures, in: Proc. ISPSD and IC's, Suisse, 1994.

[12] Z. Pavlovic, Z. Prijic, S. Dimitrijiev, N. Stojadinovic, Temperature dependence of on-resistance in low-voltage power VDMOS transistors, Microelectronics Journal 24 (1993) 115-124. 